\title{
A atuação da equipe multiprofissional na reabilitação do paciente com acidente vascular cerebral - relato de experiência
}

\author{
Júlio César das Chagas \\ Assistente Social, graduado pela Faculdade Cearense - FAC e Pós-Graduado na modalidade de Residência \\ Multiprofissional em Neurologia/Neurocirurgia pela Escola de Saúde Pública do Ceará- ESP CE \\ $\bowtie$ Juliochagas_06@yahoo.com.br
}

\begin{abstract}
Luciana Maria Nascimento da Silva
Assistente Social, Especialista em Saúde Pública e Mestre em Sociologia pela Universidade Estadual do CearáUECE. Preceptora de Núcleo na categoria de Serviço Social do Programa de Residência Multiprofissional em Neurologia/Neurocirurgia da Escola de Saúde Pública do Ceará- ESP CE.
\end{abstract}

$\square$ nascimento.n@gmail.com

\section{Resumo:}

O Acidente Vascular Cerebral (AVC) é evidenciado como uma das principais doenças que afeta a população mundial, provocando grande número de mortes e incapacidades físicas, levando o indivíduo a sofrer fortes impactos nas questões de natureza biológica, psicológica, social e econômica. O estudo aqui apesentado, trata-se de uma pesquisa descritiva, qualitativa na modalidade de relato de experiência com o objetivo de observar a atuação da equipe multiprofissional na reabilitação de pacientes acometidos com AVC na condição de internado e identificar as possíveis dificuldades existentes para atuação multiprofissional. Foi desenvolvido no período de março/2018 a março/2019, durante a atuação como profissional-residente no Programa de Residência Multiprofissional em Neurologia e Neurocirurgia da Escola de Saúde Pública do Ceará (ESP/CE), cujo cenário de prática foi um hospital terciário de Fortaleza/Ceará de referência na área. Teve como aporte metodológico a pesquisa descritiva, bibliográfica, documental, técnica de observação direta e diário de campo como instrumento de coleta de dados. Conclui-se que, a equipe multiprofissional tem um papel relevante frente à reabilitação do paciente acometido por AVC, oportunizando qualidade de vida ao usuário e uma nova chance de reinserção na sociedade, tornando-os mais independente para os seus afazeres diários após a alta hospitalar. Foram identificados desafios para o desempenho do trabalho multiprofissional, tais como fragmentação do cuidado; falhas na comunicação entre os profissionais; modelo de gestão verticalizado, dificultando assim a atuação multidisciplinar.

Palavras-chave: Acidente Vascular Cerebral, Equipe multiprofissional, Reabilitação.

\section{The role of the multiprofessional team in the rehabilitation of stroke patients - experience report}

\begin{abstract}
:
Stroke is evidenced as one of the main diseases that affect the world population, causing a large number of deaths and physical disabilities, leading the individual to suffer strong impacts on biological, psychological, social and economic issues. The present study is a qualitative descriptive research in the experience report modality with the objective of observing the performance of the multidisciplinary team in the rehabilitation of stroke patients in the condition of hospitalized and identifying the possible difficulties existing for multidisciplinary practice. It was developed from March / 2018 to March / 2019, while acting as a resident-professional in the Multiprofessional
\end{abstract}


Residency Program in Neurology and Neurosurgery of the School of Public Health of Ceará (ESP / CE), whose practice scenario was a tertiary hospital. of Fortaleza / Ceará of reference in the area. It had as methodological support the descriptive, bibliographical, documentary research, direct observation technique and field diary as data collection instrument. In conclusion, the multiprofessional team plays a relevant role in the rehabilitation of stroke patients, providing users with a better quality of life and a new chance for reintegration into society, making them more independent for their daily tasks after hospital discharge. Challenges for multiprofessional work performance were identified, such as care fragmentation; failures in communication between professionals; vertical management model, thus hindering multidisciplinary action.

Keywords: Stroke, Multiprofessional team. Rehabilitation.

\section{El papel del equipo multiprofesional en la rehabilitación de pacientes con accidente cerebrovascular: informe de experiência}

\section{Resumen:}

El accidente cerebrovascular se evidencia como una de las principales enfermedades que afectan a la población mundial, causando una gran cantidad de muertes y discapacidades físicas, lo que lleva al individuo a sufrir fuertes impactos en problemas biológicos, psicológicos, sociales y económicos. El presente estudio es una investigación descriptiva cualitativa en la modalidad de informe de experiencia con el objetivo de observar el desempeño del equipo multidisciplinario en la rehabilitación de pacientes con accidente cerebrovascular en estado de hospitalización e identificar las posibles dificultades existentes para la práctica multidisciplinaria. Fue desarrollado de marzo / 2018 a marzo / 2019, mientras actuaba como residente profesional en el Programa de Residencia Multiprofesional en Neurología y Neurocirugía de la Escuela de Salud Pública de Ceará (ESP / CE), cuyo escenario de práctica era un hospital terciario. de Fortaleza / Ceará de referencia en la zona. Tuvo como soporte metodológico la investigación descriptiva, bibliográfica, documental, la técnica de observación directa y el diario de campo como instrumento de recolección de datos. En conclusión, el equipo multiprofesional desempeña un papel relevante en la rehabilitación de pacientes con accidente cerebrovascular, brindando a los usuarios una mejor calidad de vida y una nueva oportunidad de reintegración en la sociedad, haciéndolos más independientes para sus tareas diarias después del alta hospitalaria. Se identificaron desafíos para el desempeño laboral multiprofesional, como la fragmentación de la atención; fallas en la comunicación entre profesionales; modelo de gestión vertical, lo que dificulta la acción multidisciplinaria.

Palabras clave: Accidente cerebrovascular, Equipo multiprofesional, Rehabilitación.

\section{INTRODUÇÃO}

Segundo a Organização Mundial da Saúde (2017) o Acidente Vascular Cerebral (AVC) é evidenciado como uma das principais doenças que afeta a população mundial, provocando grande número de mortes e incapacidades físicas parciais ou totais, levando o indivíduo a sofrer forte impacto nas questões de natureza psicológica, social e econômica, principalmente quando se trata de pessoas com baixa renda e de famílias em que o paciente é o provedor. Este por sua vez poderá ser acometido com o processo de perda de sua autonomia e consequentemente passará a depender de terceiros para a realização de suas atividades diárias. 
No Brasil, dados do Ministério da Saúde (2017), indicam que o AVC é o responsável por $10 \%$ dos óbitos na população adulta e consiste no diagnóstico de $10 \%$ das internações hospitalares pertencentes ao sistema público de saúde. No ano de 2016, o SUS registrou 188,2 mil internações por AVC, além de 40 mil óbitos. Sendo assim, o país apresenta a quarta posição no ranking na taxa de mortalidade por AVC entre os países da América Latina e Caribe. Esta é a principal causa de incapacidade em adultos no país, estima-se que após um ano do episódio, um quarto das pessoas foi a óbito, um quarto estão acamadas e dependentes de terceiros para realizar suas atividades básicas, e apenas um terço retornam as suas atividades diárias do cotidiano.

Dados da Secretaria de Saúde do Estado do Ceará (2015) mostram que por ano são identificados cerca de 7.249 casos de AVC no Estado, dos quais cerca de 4.500 pessoas morrem anualmente vítimas da doença, correspondendo por cerca de $10 \%$ de todos os óbitos. Atualmente a rede de saúde pública estadual conta com três unidades de referência para o tratamento da referida patologia, sendo uma em Fortaleza e outras duas no interior do Estado.

Diante da alta prevalência da doença, o Brasil tem tomado uma série de medidas para prevenção, tratamento e reabilitação destes pacientes. No entanto, tem-se efetivado legislações e linha de cuidado voltadas para esse público, destacando-se a Portaria no 1.161 / 2005 que rege a Política Nacional de Atenção ao Portador de Doença Neurológica; a Portaria nº 664/2012 que dispõe sobre o Protocolo Clínico e Diretrizes Terapêuticas e Trombólise no AVC Isquêmico Agudo e a Portaria ํㅡ 665/2012 que dispõe sobre os critérios para habilitação de Centro de Atendimento de Urgência aos pacientes com Acidente Vascular Cerebral.

No sentido de buscar o retorno desses indivíduos para uma vida social ativa, as Diretrizes de atenção à reabilitação de pessoas com Acidente Vascular Cerebral (2013), preveem que durante o processo de reabilitação deverá existir uma equipe multiprofissional com atuação interdisciplinar, composta por médico, enfermeiro, fisioterapeuta, assistente social, terapeuta ocupacional, fonoaudiólogo, psicólogo, nutricionista e farmacêutico.

A motivação para desenvolver esta pesquisa, se manifestou a partir da campanha mundial de combate ao AVC 2018, cujo tema foi "reerguendo-se após um Acidente Vascular Cerebral", criada pela Organização Mundial de AVC (WSO), que teve como foco mostrar os desafios e triunfos enfrentados pelas pessoas acometidas por essa doença. Foram diversas histórias de pacientes que passaram por processos de reabilitação, e estão reinseridos na 
sociedade, seja de forma total ou adaptada a sua nova realidade com suas limitações. A partir desta campanha foi feito um contraponto com esta pesquisa, em que é descrita a atuação destes profissionais que atuam na reabilitação objetivando o maior grau de independência nesses pacientes, considerando-os na sua integralidade, numa atitude humanizada, tendo uma abordagem ampla e resolutiva do cuidado.

Justifica-se o presente estudo a partir da experiência vivenciada como assistente social residente no Programa de Residência Multiprofissional em Neurologia e Neurocirurgia da Escola de Saúde Pública do Ceará (ESP CE), cujo cenário de prática foi um hospital terciário de referência em Fortaleza/CE, que proporcionou o contato com pacientes seqüelados devido ao AVC, marcando o local como lugar propício para a atuação multiprofissional na reabilitação destas pessoas, como também questionamentos que surgiram durante esse período: como funciona a atuação multiprofissional na reabilitação de paciente com AVC? Existem dificuldades de atuação da equipe multiprofissional? Qual papel dos profissionais frente ao paciente com AVC?

Deste modo, o estudo tem como objetivo geral: Observar a atuação da equipe multiprofissional na reabilitação de pacientes acometidos com AVC na condição de internado, tendo como objetivos específicos a) identificar as possíveis dificuldades existentes para atuação multiprofissional; b) verificar o papel da multiprofissionalidade na reabilitação dessas pessoas e c) relatar a experiência vivenciada neste cenário de prática. Bem como, apresentar a metodologia utilizada na pesquisa e a conclusão.

\section{METODOLOGIA}

Este trabalho foi desenvolvido em um Hospital Terciário de Fortaleza-CE durante o processo formativo do Programa de Residência Multiprofissional em Neurologia e Neurocirurgia pela Escola de Saúde Pública do Ceará, cujo período da pesquisa foi compreendido entre março de 2018 a março de 2019. Este hospital é referência para o Norte e Nordeste devido a assistência de alta complexidade, sendo o maior hospital público da rede 
estadual, realizando neurocirurgias, prestando assistência ao AVC e outras doenças neurológicas.

A metodologia escolhida foi de natureza eminentemente qualitativa, a qual segundo Minayo (2001, p. 22) trabalha com o universo de significados, motivos, aspirações, crenças, valores e atitudes, o que corresponde a um espaço mais profundo das relações, dos processos e dos fenômenos que não podem ser reduzidos à operacionalização de variáveis.

Trata-se de uma pesquisa descritiva qualitativa na modalidade de relato de experiência, definido como um texto que descreve precisamente uma experiência vivenciada que possa contribuir de forma relevante para sua área de atuação. Ele traz as motivações e as metodologias para as ações tomadas na situação e as considerações que a vivencia trouxe aquele que a viveu (ABNT 6023, 2002).

Para nortear a pesquisa foram definidas as seguintes categorias de análise: Acidente vascular cerebral; equipe multiprofissional e a reabilitação do paciente com AVC. Foi então, utilizada no primeiro momento a pesquisa bibliográfica para ter uma base de fundamentação teórica, utilizando como fontes de pesquisa bancos de dados como Biblioteca Virtual em Saúde (BVS), Scielo, Rede Brasil de AVC, revistas, livros, artigos acadêmicos, acessados no período de março de 2018 a março de 2019.

Gil (2002, p.44) argumenta que a pesquisa bibliográfica é desenvolvida com base em material já elaborado, construído principalmente de livros e artigos científicos, na qual foi utilizada fundamentalmente as contribuições dos diversos autores sobre o tema em foco. Deste modo, foram utilizados autores vinculados à área, tais como Andrade (2013), Spence (2013), Saraiva (2017) e Peduzzi (2001).

Utilizou-se ainda a pesquisa documental com a finalidade de fundamentar as observações, tomado como base o tipo de atendimento a ser realizado e que profissionais são recomendados legalmente para esse perfil de paciente. Neste sentido, Gil (2002, p.45) define como sendo a realização de análises de materiais que não receberam ainda um tratamento analítico, ou que ainda podem ser reelaborados de acordo com os objetos da pesquisa, são documentos considerados fontes ricas e estáveis de dados, já que subsistem ao longo do tempo, tornam-se a mais importante fonte de dados em qualquer pesquisa de natureza histórica. 
Para tanto, foram consultadas as Portarias do Ministério da Saúde que efetivam as políticas de prevenção, tratamento e reabilitação de pessoas com AVC, tais como a Portaria Gabinete do Ministro/MS nº 1.161/ 2005 que rege a Política Nacional de Atenção ao Portador de Doença Neurológica; Portaria Gabinete do Ministro/MS nº 664/2012 que dispõe sobre o Protocolo Clínico e Diretrizes Terapêuticas e Trombólise no AVC Isquêmico Agudo; Portaria Gabinete do Ministro/MS nº665/2012 que dispõe sobre os critérios para habilitação de Centro de Atendimento de Urgência aos pacientes com Acidente Vascular Cerebral; e também documentos examinados do Ministério da Saúde como o Manual de Rotina para Atenção ao AVC (2013); Diretrizes de Atenção à Reabilitação de Pessoas com Acidente Vascular Cerebral (2013) e a Linha de Cuidados em Acidente Vascular Cerebral (2012).

Para o processo de coleta de dados foi utilizada a técnica da observação direta, que segundo Marconi e Lakatos (2003, p.190), trata-se de uma técnica de coleta com a finalidade de conseguir informações e utilizando os sentidos na obtenção de determinados aspectos da realidade. Não consiste apenas em ver e ouvir, mas também em examinar fatos ou fenômenos que se desejam estudar, podendo ser através de entrevista e observação.

Deste modo, foi realizada a técnica no processo de investigação durante os rodízios nas unidades do hospital, na Unidade de Acidente Vascular Cerebral (UAVC) (março a maio/2018); Enfermaria Neurológica (junho a agosto/2018); Enfermaria Neurocirúrgica (setembro a outubro/2018); Unidade de Terapia Intensiva Neurológica (janeiro a março/2019). Tendo nessas unidades equipes compostas por profissionais da Enfermagem, Farmácia, Fisioterapia, Fonoaudiologia, Nutrição, Psicologia, Serviço Social, Medicina, Terapia Ocupacional e Técnicos / Auxiliares de Enfermagem.

Como instrumento de coleta, foi utilizado também o Diário de Campo que nas palavras de Lewgoy, Scavoni (2002, p.63) é um documento pessoal-profissional no qual o estudante [profissional] fundamenta o conhecimento teórico-prático, relacionando com a realidade vivenciada no cotidiano profissional, através do relato de suas experiências e sua participação na vida social.

Desta forma, o referido instrumental foi elaborado com o objetivo de ter elementos para realizar as análises da pesquisa. Foi organizado primeiramente com a anotação dos fatos e fenômenos sociais encontrados durante a atuação profissional, por exemplo, como era 
realizado o atendimento aos pacientes, qual tipo de profissional estava evolvido, se havia uma multiprofissionalidade naquele atendimento ou no decorrer da intenção dos pacientes. No segundo momento foram realizados registros para as interpretações do que havia sido anotado. É importante salientar que havia a preocupação de fazer as anotações no momento em que as observações eram realizadas na Unidade, para que não ocorresse a deturpação dos fatos, a partir de esquecimentos de pequenos detalhes.

\section{RESULTADOS}

Vivenciar a atuação de uma equipe multiprofissional requer inicialmente que os sujeitos envolvidos compreendam o significado do trabalho em grupo, para que todos os saberes sejam alinhados e voltados para o bem-estar do paciente, é compreender que a questão saúde-doença vai além dos fatores biológicos, devendo ser considerado fatores determinantes e condicionantes, tais como questões sociais, ambientais, econômicas, psicológicas entre outras.

Referindo-se a experiência vivenciada, a atuação da equipe multiprofissional se dava inicialmente no momento da internação, onde cada profissional Enfermeiro, Médico, Fisioterapeuta, Fonoaudiólogo, Farmacêutico, Nutricionista, Terapeuta Ocupacional faziam as suas avaliações específicas de forma uniprofissional.

A equipe da Unidade após a avaliação clínica inicial encaminhava o familiar/acompanhante ao profissional do Serviço Social para que fosse realizada uma abordagem socioeconômica, começando com uma entrevista inicial, na qual o assistente social utilizava a Ficha de Acompanhamento Social (FAS) como instrumento de coleta de dados para subsidiar o atendimento, a fim de compreender a realidade que aquele paciente estava inserido, identificando assim as possíveis expressões da questão social e fazer as devidas orientações e encaminhamentos necessários durante o período da internação na Unidade. Nesta perspectiva, pode-se destacar nesse estudo, o caso de um paciente conforme narrativa: 
Compreendendo a importância da atuação multiprofissional na reabilitação da pessoa acometida com AVC, é relatado um caso que marcou a passagem por esse período de vivência. Trata-se de uma pessoa do sexo masculino, 60 anos de idade, solteiro, agricultor, hipertenso, tabagista e etilista pesado, que deu entrada na emergência neurológica apresentando Acidente Vascular Cerebral isquêmico (AVCI) em grande extensão, fora de janela para Trombólise e evoluindo para tetraplegia, sendo realizado procedimento de intubação orotraqueal, sonda nasoenteral e vesical no mesmo dia da internação. (OBSERVAÇõES DO PESQUISADOR, 2018).

O referido paciente teve um período de internação de quatro meses, de março a junho/ 2018, sendo considerado perfil crítico-crônico, passando por diversas intervenções da equipe multiprofissional.

A equipe de Fisioterapia realizava exercícios e procedimentos de reabilitação pulmonar, objetivando o desmame da ventilação mecânica. Depois de perceber a melhora clínica do paciente ao longo do tempo e estando hemodinamicamente estável, foi realizado a extubação, retirada a sedoanalgesia e feito a traqueostomia pela equipe médica. Tão logo, o paciente passou a compreender alguns comandos de forma limitada, respondendo com o piscar de olhos e alguns movimentos da cabeça.

Neste momento é importante salientar a atuação dos profissionais da Terapia Ocupacional, que desenvolveram técnicas de comunicação com esse paciente através de comandos dos olhos e cabeça, no qual se fazia perguntas para obter respostas através destes movimentos. Essa técnica foi demostrada para os familiares que faziam seu acompanhamento diariamente e passaram a ter essa forma de contato com seu familiar. Portanto, o trabalho desenvolvido pelos profissionais de saúde da unidade ficou evidente:

Os profissionais da Fisioterapia desenvolveram técnicas para sentá-lo na beira do leito apoiado em uma cadeira confeccionada artesanalmente com tubos de PVC, para realizar os exercícios recomendados para a situação, assim como evitar complicações decorrentes de longos períodos na mesma posição. A Fonoaudiologia atuou fazendo terapias com o objetivo de ajustar as condições clínicas que impediam a ingestão de alimento seguro por via oral, mas não houve respostas suficientes, permanecendo em uso da sonda de nasoenteral (OBSERVAÇÕES DO PESQUISADOR, 2018). 
Assim sendo, foi imprescindível a atenção integrada à saúde do paciente com AVC que propõe uma abordagem multi/interdisciplinar, que contemplou a programação de reuniões periódicas dos profissionais para a discussão de cada caso, incluindo as estratégias de atendimento e plano terapêutico abordado. Dessa forma, a família do paciente pode receber orientações uniformes e consensuadas pela equipe envolvida no tratamento e reabilitação, o que facilitou a adesão ao tratamento.

Por conseguinte, para além destas ações ocorreram outras iniciativas importantes da equipe tendo como foco o paciente:

Houve intervenções dos profissionais da Psicologia, se utilizando de técnicas de comunicação em consonância com os profissionais da Fisioterapia e Terapia Ocupacional. Assim como, acompanhamento dos familiares que se encontravam fragilizados com a situação de seu ente. Houve reuniões com equipe multiprofissional para repassar a situação de saúde do paciente, os planos terapêuticos, o que havia avançado no tratamento e também a identificação dos desafios no momento. A equipe de Serviço Social atuou nas demandas sociais que surgiram nesta fase, tais como orientações para familiares acerca do AuxílioDoença junto ao Instituto Nacional do Seguro Social (INSS), processo de Curatela junto a Defensoria Pública Estadual, assim como a avaliação das condições sociais, incluindo o apoio da família e da rede sócio - assistencial de serviços públicos disponíveis, como também na aquisição de insumos contribuindo assim para o processo de desospitalização (OBSERVAÇÕES DO PESQUISADOR, 2018).

Durante as atuações em campo, foi possível identificar o papel e a relevância da equipe multiprofissional, colocando em prática a Clínica Ampliada e o Projeto Terapêutico Singular (PTS).

A proposta de Clínica Ampliada é destinada a todos os profissionais da saúde que trabalham e fazem clínica. Toda profissão faz um recorte, um destaque de sintomas e informações, cada uma de acordo com seu núcleo profissional. Ampliar a clínica significa justamente conciliar os recortes teóricos de cada profissão às necessidades dos usuários. (BRASIL, 2010, p. 25). Preconizada pelo SUS, recuperando tal indivíduo para reinseri-lo na sociedade de forma mais independente possível, observando a integralidade do sujeito e alinhando os saberes da equipe. 
Complementando a proposta da clínica ampliada, havia na unidade semanalmente a realização da visita multiprofissional leito a leito com toda a equipe envolvida na terapêutica do paciente. Neste momento era repassado a situação clínica de tal indivíduo e cada profissional dava sua contribuição quanto às suas intervenções e o histórico do paciente. Dessa forma também havia a tentativa de implementação de um Projeto Terapêutico Singular (PTS) nas unidades, tratando-se de um conjunto de propostas terapêuticas articuladas, para um sujeito individual, resultado da discussão coletiva de uma equipe multi/interdisciplinar (BRASIL, 2010, p. 27). Neste momento foi possível observar um dos pontos frágeis na equipe, na qual o modelo biomédico instalado na instituição, se sobressaia sobre as opiniões de outros profissionais da saúde não médico.

Com o propósito de dar continuidade ao processo de reabilitação, a equipe preparava paulatinamente o paciente e os familiares para sua nova realidade. Em alguns momentos eram realizados atendimentos conjuntos com os profissionais da Psicologia e Serviço Social, Fisioterapia e Terapia Ocupacional, Nutrição e Fonoaudiologia, Farmácia e Enfermagem e outros profissionais envolvidos na terapêutica. É importante salientar que esses momentos ocorriam quando a situação do paciente ultrapassava as condições e as intervenções uni profissionais, não sendo algo de rotina na tentativa de trabalhar uma equipe multi e interprofissional conectada.

Em virtude do trabalho desenvolvido na unidade com a equipe multiprofissional no sentido de favorecer um processo de desospitalização bem-sucedido salienta-se:

O referido paciente estando em situação clínica para alta hospitalar, foi iniciado o processo de desospitalização junto a família, realizando reuniões familiares para explicar como seria essa nova realidade familiar, quais os cuidados necessários, a partir deste momento foi realizado treinamento com os familiares focando os cuidados em saúde. A equipe da Nutrição orientou quanto a formulação de dieta artesanal, assim como os cuidados com a sonda nasoenteral e a alimentação, lhes fornecendo manual de orientações alimentares. Quanto a cadeira artesanal de PVC citada anteriormente, a família buscou orientações com a equipe de fisioterapeutas para realizar a confecção em seu lar (OBSERVAÇÕES DO PESQUISADOR, 2018). 
Na experiência vivenciada é notório que a soma de práticas e saberes foram utilizadas de maneira consonante buscando de forma diversa, dentro das possibilidades a mobilização de toda a equipe multiprofissional em prol da reabilitação do paciente com AVC:

Os profissionais do Serviço Social e Médicos da unidade hospitalar fizeram a contrareferência para o município de origem do paciente solicitando serviços de terapias multiprofissional no domicílio, assim como, solicitação de insumos hospitalares juntos aos órgãos públicos, como cama e colchão hospitalar, cadeira de rodas, fraldas e dieta. A enfermagem realizou as orientações quanto aos cuidados com a lesão por pressão. Este paciente recebeu alta domiciliar estando tetraplégico, consciente, contactuante, interagindo bem e obedecendo a comandos, com o traqueostomo de metal e respirando em ar ambiente. Desta forma, percebe-se que os esforços e os diversos saberes profissionais contribuíram de forma consistente para o processo de reabilitação desse paciente e que o processo saúde-doença vai para além da questão biológica (OBSERVAÇÕES DO PESQUISADOR, 2018).

No tocante a preocupação com o paciente em pós-alta hospitalar, a articulação para a continuação desse tratamento fora da unidade, se deu, com a participação da equipe de Serviço Social, no qual articulou com a rede de serviços públicos os encaminhamentos necessários deste indivíduo por meio de solicitação da equipe médica. Em outros momentos foi possível o contato com os profissionais de Fonoaudiologia e Fisioterapia para que estes orientassem estratégias de se conseguir tratamentos adequados quando ocorresse dificuldade de atendimento na rede SUS, como por exemplo, orientações para o atendimento em clínicas escola de reabilitação das universidades e faculdades locais que prestam serviços gratuitos a comunidade.

Considerando a relação da equipe multiprofissional com familiares e acompanhantes, pode-se elencar a realização de um grupo de Educação em Saúde, no qual se constituía de roda de conversa fomentado pela Terapia Ocupacional e Serviço Social, realizado quinzenalmente que trabalha aspectos do AVC, tais como prevenção, tratamento e reabilitação.

Importante salientar que não havia participação dos demais profissionais da equipe multiprofissional nesse grupo de Educação em Saúde, embora se tenha convidado os esses 
profissionais. Fazendo uma análise sobre este momento, percebeu-se que havia um desinteresse de algumas categorias está inserido nesses espaços; a questão do grande volume de trabalho nas unidades para uma pequena quantidade de profissionais; problemas na escala de alguns profissionais que não estavam disponíveis no momento no evento; a falta de empatia que alguns profissionais tinham para trabalhar com familiares, tornando-se profissionais mais tecnicistas, devido a sua formação.

Verificou-se que no momento da equipe do referido grupo com os familiares, surgiram muitas dúvidas quanto a essa patologia, assim como anseios e preocupações de como seria esse paciente pós-alta, devendo-se as sequelas que haviam adquiridos depois do evento. Mas havia também um distanciamento de outras categorias profissionais referente a esse grupo, o que sobrecarregava os profissionais envolvidos, contribuindo diretamente para o enfraquecimento dessas ações.

Durante essa vivência foram identificadas barreiras para o desemprenho do trabalho multiprofissional, tais como imprecisões no trabalho; fragmentação do cuidado; falhas na comunicação entre os profissionais, pois os saberes especializados se resguardam em cada profissão; estruturas implantadas nas redes hospitalares; modelo de gestão verticalizado que apresentam uma oferta de assistência impessoal e fragmentada, partindo para a indefinição de vínculos entre usuários e profissionais, dificultando essa atuação multidisciplinar.

\section{DISCUSSÃO}

\section{A precarização da saúde e o Acidente Vascular Cerebral}

Compreende-se que o Sistema Único de Saúde (SUS) foi uma grande conquista para a população brasileira, tendo como objetivo a garantia do acesso igualitário e universal aos diferentes níveis de atenção, baseado pelos princípios da universalidade, integralidade, equidade, regionalização e descentralização, este, vem enfrentando diversos desafios na contemporaneidade intensificados pela atual conjuntura do país.

Rosemberg (2013) argumenta que a saúde pública brasileira vem sofrendo grandes ameaças nos últimos tempos, sendo traduzidas em enormes filas nas unidades de saúde, 
deficiência de leitos hospitalares, falta de equipamentos e insumos básicos para seus procedimentos, escassez de recursos, baixos valores pagos pelos procedimentos realizados, precarização do trabalho dos profissionais da saúde e etc. No entanto, essas situações que os usuários vivenciam, são reflexos do plano de desmonte da saúde projetado para mercantilização e privatização, ao governo neoliberalista que se instalou no pais na última década. Isso se percebe pela falta de prioridade e pela a redução dos investimentos do poder público nesta área, reforçados pela Emenda Constitucional 95 (EC-95/2016) que congela o orçamento público durante vinte anos, justificado pela "crise econômica" que perpassa o país.

O processo de precarização e o desmonte na política de saúde brasileira põe em risco a garantia dos cidadãos nos processos de prevenção, tratamento e reabilitação principalmente nas patologias que possuem um alto índice de acometimento da população. Encontra-se nesta condição o AVC no qual a Organização Mundial da Saúde (OMS, 2017) aponta como uma das principais doenças que afeta a população mundial, causando grande número de mortes e incapacidade física nos indivíduos afetados, provocando impactos sociais, econômicos e previdenciários. São fatores que podem modificar radicalmente a rotina de familiares e pacientes afetados por tal enfermidade, principalmente em países subdesenvolvidos em que o acesso ao tratamento é precário, as condições socioeconômicas dos indivíduos de baixa renda são insuficientes e os impedem de ter condições de atendimento adequado para acolhimento do paciente e familiar que passarão a conviver com tais sequelas diariamente.

Dados do Ministério da Saúde (2017), apontam que o Brasil é apresenta a quarta posição no ranking na taxa de mortalidade por AVC entre os países da América Latina e Caribe. Esta é a principal causa de incapacidade em adultos no país, estima-se que após um ano do episódio, um quarto das pessoas foi a óbito, um quarto estão acamadas e dependentes de terceiros para realizar suas atividades básicas, e apenas um terço retornam as suas atividades diárias do cotidiano.

Oliveira (2016) define o AVC de como sendo uma doença cerebrovascular, que ocorre devido a alterações na circulação sanguínea do cérebro, havendo uma interrupção de oxigênio nesta região causando a morte das células. Pode-se classificar em dois tipos, o primeiro é o Acidente Vascular Cerebral Isquêmico (AVCI) que ocorre quando há a formação de trombos nos vasos sanguíneos cerebrais, causando a interrupção da passagem sanguíneo 
para o cérebro, que segundo Buchan, Balami e Arba (2013) corresponde há cerca de 67 a 80\% dos casos; e o segundo é Acidente Vascular Cerebral Hemorrágico (AVCH), que ocorre devido a ruptura de um vaso sanguíneo dentro do cérebro (hemorragia intracerebral) ou na membrana entre o cérebro e o crânio (hemorragia subaracnóide), este estando associado a altas taxas de mortalidade em comparação ao primeiro.

Sobre os fatores de riscos, Cancela (2008) afirma que a hipertensão arterial, o tabagismo, o colesterol elevado, o alcoolismo, o diabetes, a obesidade, doenças cardíacas, a hereditariedade, o sedentarismo, o uso de anticoncepcionais orais e a idade são fatores que podem contribuir para um evento futuro. No entanto, para que se possa prevenir, é importante salientar que estes fatores podem ser modificados a partir de mudanças de hábitos, no estilo de vida ou realização de acompanhamento médico.

No que se refere às manifestações clínicas, busca-se referenciamento nas Diretrizes de Atenção à Reabilitação à Pessoa com Acidente Vascular Cerebral (2013), que apresenta como sintomatologia os sinais de diminuição da sensibilidade, fraqueza unilateral que tenha iniciado de forma súbita na face, nos braços, nas pernas; confusão mental, dificuldade para falar ou para compreender o que é dito; alterações visuais em um ou em ambos os olhos; dificuldade para andar, perda de equilíbrio e/ou da coordenação motora; dor de cabeça intensa sem causa aparente e dormência unilateral em membros do corpo.

Neste sentido, as possíveis sequelas podem ter um caráter permanente ou temporário, sendo necessário perpassar pelo cuidado continuo de uma equipe multiprofissional durante o processo de reabilitação. O referido documento do Ministério da Saúde (2013), diz que estas sequelas podem ser déficit sensório-motor como disfagia (dificuldade de deglutição); paralisia facial que se caracteriza pela diminuição dos movimentos faciais, com consequente impacto estético e funcional; fraqueza muscular, sendo esta uma das maiores contribuintes para a incapacidade após AVC; déficits de sensibilidade, alterando capacidade sensitivas tais como dor, tato e sensação térmica; alterações visuais, que podem variar de leve a grave; limitações de atividades motoras e funcionais. 


\section{A atuação da equipe multiprofissional no AVC}

Preconiza-se que o processo de reabilitação do paciente com AVC, seja desenvolvido por profissionais com base na multidisciplinariedade e interdisciplinaridade, integrados aos princípios do SUS como a universalidade, equidade e integralidade, avaliando o paciente como ser integral nos seus diferentes aspectos sociais, biológicos, psicológicos, culturais e econômicos.

A compreensão de que a saúde vai para além dos processos biológicos, foi reformulada a partir das definições da Organização Mundial de Saúde (OMS) em 1948, em que o conceito de saúde foi definido como o completo bem-estar físico, mental e social. Essa definição se amplia no contexto mundial, como também se atualiza pela a criação de normas legais brasileiras, que a partir da Lei 8.080/90 regulamentam o Sistema Único de Saúde (SUS) e traz em seu Art. $3^{\circ}$ que a saúde tem seus fatores determinantes e condicionantes, a moradia, o trabalho, a renda, a educação, o transporte, o lazer, o saneamento básico, o meio ambiente e acesso a bens e serviços essenciais.

Quanto ao contexto histórico do trabalho multiprofissional, este veio se firmar com o avanço da ciência e surgimento de novas doenças, onde a área da saúde buscou cada vez mais a participação de diversos profissionais, a fim de compartilhar e interagir seus conhecimentos. Saraiva apud Veloso (2015) afirma o profissional médico dominavam todo o conhecimento e exercia todas as ações, com seu modelo curativista e hospitalocêntrico. Nos tempos atuais é impossível que apenas um profissional exerça o conjunto amplo e complexo das ações na área da saúde, surgindo a necessidade para a formação equipes multiprofissionais nos espaços de trabalho.

Compreende-se esta atuação multiprofissional como uma inter-relação entre os diferentes profissionais, que considera o paciente na sua integralidade, numa atitude humanizada, tendo uma abordagem ampla e resolutiva do cuidado. Previatti, Lobo e Pereira apud Minayo (2013) afirmam que a atuação da equipe se consiste em uma articulação entre várias disciplinas tendo como foco o objeto, o problema ou tema complexo, para qual não basta a resposta de uma só área. Trata-se, portanto, de uma estratégia para compreender, interpretar e explicar temas complexos dos usuários dos serviços. 
Em pacientes acometidos com AVC, se faz necessário esta abordagem multiprofissional e interprofissional, ampliando o sucesso na reabilitação, já que uma única especialidade profissional não conseguirá dar resposta a diversos fatores associados a situações de doença, hospitalização e reabilitação. Para isto as Diretrizes de atenção à reabilitação de pessoas com Acidente Vascular Cerebral (2013) recomendam que estas equipes sejam compostas por profissionais médicos, enfermeiros, fisioterapeutas, fonoaudiólogos, terapeutas ocupacionais, farmacêuticos, assistentes sociais, psicólogos, nutricionistas, auxiliares e técnicos de enfermagem, atuando de forma multi e interprofissional. Cardoso (2015 apud Saraiva, 2010) refere que é impossível fazer investigação clínica ou tratar doentes sem recorrer ao trabalho integrado de equipes multiprofissionais.

Apesar dos dispositivos legais que formalizam a atuação multiprofissional na rede de saúde pública, percebe-se na prática a existência de desafios para esta atuação em âmbitos hospitalares, devido às estruturas implantadas nas redes hospitalares, seguidos por modelo de gestão verticalizado, que apresentam uma oferta de assistência impessoal e fragmentada, partindo para a indefinição de vínculos entre usuários e profissionais. Araújo et al (2016) afirmam que extrapolar a lógica do trabalho uniprofissional, no Brasil, ainda é um desafio, pois trabalhar no mesmo ambiente e estar junto em uma atividade ou ação não significa efetivar a atuação multi e interprofissional. Desta forma, se faz necessário adotar a interprofissionalidade e multiprofissionalidade como estratégia de trabalho e de formação para a reabilitação do paciente com AVC.

\section{A reabilitação do paciente pós - AVC}

Atualmente, o modelo hegemônico de reabilitação brasileiro é baseado nas instituições Centros de Reabilitação, onde esses procedimentos concentram-se nos centros apropriados para tais serviços. No entanto, reconhecer o atual cenário da reabilitação no país é ter a necessidade de que o acesso perpassou por um longo período histórico até a atual conjuntura. Almeida e Campos (2002), dizem que as primeiras ideias de reabilitação apareceram no Brasil na década de 1950 com a implantação do Instituto Nacional de Reabilitação (INR) no Estado de São Paulo, dotado de materiais especiais e equipe multiprofissional. Com a Constituição Federal de 1988, e a partir dos anos 1990 com a criação 
da Lei Orgânica da Saúde, o SUS nasceu com a ideia inicial de fortalecimento da atenção integral a saúde, com ênfase nas ações de promoção, reabilitação e inclusão social. $\mathrm{Na}$ convenção sobre os direitos das pessoas com deficiência (2012) recomenda-se que os estados deverão adotar estratégias para fortalecimento e ampliação de programas de reabilitação nas áreas da saúde, possibilitando a autonomia dos indivíduos deficientes.

Discutir a definição de reabilitação é algo complexo, tendo em vista as inúmeras publicações sobre a temática, para tanto, apresenta-se o conceito de Ribeiro e Ribeiro (2010), que diz:

\begin{abstract}
O conceito de reabilitar inclui diagnóstico, intervenção precoce, uso adequado de recursos tecnológicos, continuidade de atenção e diversidade de modalidades de atendimentos visando à compensação da perda da funcionalidade do indivíduo, à melhoria ou manutenção da qualidade de vida e à inclusão social [...] o trabalho de reabilitação deve englobar tanto o aspecto técnico quanto o aspecto da cidadania do indivíduo com deficiência, que tem o direito de fazer escolhas e de ser o autor de sua própria história. (RIBEIRO; RIBEIRO et al. 2010).
\end{abstract}

Cancela (2008) afirma que esse momento deve-se iniciar na fase aguda da doença e ainda no âmbito hospitalar, assim que o paciente adquirir estabilidade hemodinâmica consiste em técnicas aplicadas por uma equipe multiprofissional, contribuindo para recuperar o mais breve a função anterior do indivíduo, tornando-o mais independente possível, com essas terapias a tendência é o desaparecimento ou melhoras destas sequelas. Devido à capacidade do cérebro em aprender e mudar, fazendo com que ocorra a neuroplasticidade, onde as células de outras áreas do cérebro, que não foram atingidas pelo AVC, assumem funções realizadas pelas células da área afetada. E em seus aspectos gerais a terapêutica do AVC compreende-se entre a prevenção, o tratamento agudo e a reabilitação.

Ser acometido por um AVC é algo que pode modificar a rotina do paciente completamente, assim como de seus familiares. Para além das sequelas motoras, esse indivíduo pode desenvolver transtornos psicológicos, como a depressão. Poderá apresentar expressões da questão social que impossibilite o acolhimento deste paciente em seu domicilio, como por exemplo, a estrutura inadequada do ambiente domiciliar, pouco ou 
nenhum apoio familiar, poucos recursos financeiros para custear gastos necessários e assim, dificultar o acesso ao processo de reabilitação.

Para dar início a esta etapa, é necessário a presença de alguns fatores, tais como citados por Teasell (2013) o paciente deve esta hemodinamicamente estável; capaz de aprender; ter resistência física suficiente para participar ativamente das terapias e capacidade de sentar sem suporte em uma cadeira de rodas por pelo menos uma hora de cada vez. Deve-se ainda constar atividades que possam ser realizadas em qualquer meio que represente situações do cotidiano destes pacientes, onde seja possível concentrar-se, interagir, tomar decisões e expressar seus sentimentos.

O acesso à continuidade do tratamento referente a reabilitação, com qualidade e com participação de equipe multiprofissional é um direito que assiste a todos os cidadãos brasileiros, existindo dispositivos legais para sua garantia, tais como a Carta dos Direitos dos Usuários da Saúde (2012) afirmando que é um direito da pessoa ter atendimento adequado, com qualidade no tempo certo e com garantia de continuidade do tratamento, para isso deve ser assegurado o atendimento ágil, com tecnologia apropriada, por equipe multiprofissional capacitada e com condições adequadas de atendimento.

\section{CONCLUSÃo}

A partir dos dados epidemiológicos expostos e da experiência vivenciada nas Unidades citadas, pode-se constatar a grande quantidade de pessoas que são acometidas pelo acidente vascular cerebral diariamente, e que esta patologia afeta não somente os aspectos biológicos, mas também todo um contexto psicossocial que envolvem pacientes e familiares, exigindo intervenção multiprofissional nos diversos aspectos que envolvem o adoecimento humano.

Com as abordagens metodológicas realizadas nesta pesquisa, pode-se concluir que foram alcançados os objetivos propostos, demonstrado através de relato de experiência a atuação da equipe multiprofissional destacando sua relevância nesse processo de reabilitação, oportunizando qualidade de vida aos usuários e uma nova chance de reinserção 
na sociedade, tornando-os mais independente para os seus afazeres diários após a alta hospitalar.

Verificada a variedade de profissionais e de saberes, os diversos dispositivos que reafirmam a importância dessa atuação em equipe, pode-se compreender que essa foi uma grande conquista para a saúde brasileira e para o usuário em especial, tendo em vista que descentralizou essas atividades para outros profissionais com conhecimentos específicos, criando possibilidades para a desconstrução do modelo de saúde uniprofissional e medicalocêntrico.

Com esse estudo, foram identificadas algumas barreiras para o desemprenho do trabalho multiprofissional, tais como imprecisões no trabalho; fragmentação do cuidado; falhas na comunicação entre os profissionais, pois os saberes especializados se resguardam em cada profissão; estruturas implantadas nas redes hospitalares; modelo de gestão verticalizado que apresentam uma oferta de assistência impessoal e fragmentada, partindo para a indefinição de vínculos entre usuários e profissionais, dificultando essa atuação multidisciplinar.

Importante salientar que para alguns profissionais da equipe multiprofissional, tenhase percebido a falta de empatia para trabalhar com outro, onde as características tecnicistas predominavam em suas atuações, deixando de ver o paciente na sua integralidade.

Considerando o ponto de vista pessoal, a experiência no Programa de Residência Multiprofissional proporcionou grande crescimento no sentido de ampliar horizontes tanto no aspecto profissional quanto pessoal. E salientar ainda que esse estudo possa contribuir de modo consistente na a elaboração de futuras pesquisas.

Desta forma, podem-se construir conhecimentos específicos na área da neurologia/ neurocirurgia e da atuação multiprofissional em saúde, gerando uma aproximação com as demais categorias e podendo visualizar os entraves e conquistas para essa atuação multiprofissional no cotidiano. 


\section{REFERÊNCIAS}

ALMEIDA, M. C. de.; CAMPOS, G. W. S. Políticas e modelos assistenciais em saúde e reabilitação de pessoas com deficiência no Brasil: análise de proposições desenvolvidas nas últimas duas décadas. Rev. Ter. Ocup. Univ. São Paulo, v. 13, n. 3, p. 118-26, set. /dez. 2002.

ASSOCIAÇÃO BRASILEIRA DE NORMAS TÉCNICAS. NBR 6023: Informação e documentação: Referências. Rio de Janeiro, 2002.

BRASIL. Constituição da República Federativa do Brasil de 1988. Disponível em http://www.planalto.gov.br/ccivil_03/constituicao/constituicaocompilado.htm. Acesso em: 05 out. 2017.

Carta dos Direitos dos usuários da Saúde. $1^{\circ}$ ed. Brasília, DF: Ministério da Saúde/ Conselho Nacional de Saúde, 2012.

Convenção sobre os Direitos das Pessoas com Deficiência: Protocolo Facultativo à Convenção sobre os Direitos das Pessoas com Deficiência: Decreto Legislativo no 186, de 09 de julho de 2008: Decreto $n^{\circ}$ 6.949, de 25 de agosto de 2009. 4.ed., rev. e atual. Brasília-DF: Secretaria de Direitos Humanos, 2010.

Ministério da Saúde. AVC: o que é, causas, sintomas, tratamentos, diagnóstico e prevenção. Disponível em: http://portalms.saude.gov.br/saude-de-a-z/acidente-vascular-cerebral-avc. Acesso em: 22 out. 2018.

Secretaria de Atenção à Saúde. Departamento de Ações Programáticas Estratégicas. Diretrizes de atenção à reabilitação da pessoa com acidente vascular cerebral. Brasília, DF, 2013.

. Caderno de Atenção Básica: Diretrizes do NASF: Núcleo de Apoio a Saúde da Família / Ministério da Saúde, Secretaria de Atenção à Saúde, Departamento de Atenção Básica. Brasília: MS, 2010.

. MINISTÉRIO DA SAÚDE. Lei nº 8.080. Lei Orgânica da Saúde de 19 de setembro de 1990.

BUCHAN, Alastair M, BALAMI, Joyce S, ARBA, Francesco. Epidemiologia da prevenção do acidente vascular cerebral e urgência do tratamento. In: SPENCER, J.; BARNETT,HENRY. Acidente vascular cerebral: prevenção, tratamento e reabilitação. Tradução BURNIER, Jussara N.T. Porto Alegre, RS: Artmed, 2013.

CANCELA, Diana M. G.. O Acidente vascular cerebral- classificação: principais consequências e reabilitação. 2008. Disponível em: www.psicologia.com.pt. Acesso em: 01 nov. 2017.

CEARÁ. Secretária de Saúde do Estado do Ceará. Prevenção diminui em 90\% o risco de AVC. Disponível em: http://www.ceara.gov.br/2017/10/27/prevencao-diminui-em-90-o-risco-de-avc/. Acesso em: 23 out. 2018.

GIL, Antônio Carlos. Como elaborar projetos de pesquisa. 4. ed. São Paulo: Atlas, 2002.

LEWGOY, Alzira M. B; SCAVONI, Maria Lucia. Supervisão em Serviço Social: a formação do olhar ampliado. In: Revista Texto \& Contextos. EDIPUCRS. Porto Alegre: 2002.

MARCONI, Marina de Andrade; LAKATOS, Eva Maria. Fundamentos da metodologia cientifica. 5. ed. São Paulo: Atlas, 2003. 
MINAYO, Maria Cecília de Souza (Org.). Pesquisa social: teoria, método e criatividade. 18 ed. Petrópolis, RJ: Vozes, 2001.

OLIVEIRA, Edênia S. G. Como reconhecer o Acidente Vascular Cerebral- AVC. Rede de Teleassistência de Minas Gerais. Minas Gerais, 2016.

PREVIATTI, Débora. LOBO, Eduardo. PEREIRA, Juliana. Em busca da Interdisciplinaridade: o trabalho multiprofissional na gestão pública em saúde para a construção do Sistema Único de Saúde (SUS). In: Coleção Gestão de Saúde Pública, v. 1. ed. Fundação Boiteux. Florianópolis,SC, 2013.

REDE BRASIL DE AVC. Campanha AVC 2017. Disponível em: http://www.redebrasilavc.org.br/campanha-avc2017/. Acessado em: 21 out. 2018.

RIBEIRO, CTM, Ribeiro MG, Araújo AP, Mello LR, Rubim LC, Ferreira JES. O sistema público de saúde e as ações de reabilitação no Brasil. Revista Panam Salud Publica, v.28, nํ1, p. 43-8. 2010.

ROSEMBERG, Ana Margarida Furtado Arruda. Breve Histórico da Saúde Pública no Brasil. In: ROUQUAYROL, M. Z.; GURGEL, M. (Org.). Epidemiologia e Saúde. Rio de Janeiro: Med Book, 2013.

SARAIVA, Maria R. F. A importância das equipes multidisciplinares. Disponível em: http://www.atlasdasaude.pt/publico/content/importancia-das-equipas-multidisciplinares. Acesso em: 27 out. 2017.

SILVA, Emanuel de Jesus Alves. Reabilitação após AVC. 2010. Dissertação (Mestrado Integral-Medicina) Faculdade de Medicina, Universidade de Porto. Porto/ Portugal, 2010.

SPENCE, J. David. BERNETT, Henry J.M. Acidente vascular cerebral: prevenção, tratamento e reabilitação. Tradução BURNIER, Jussara N.T. Ed. Artmed. Porto Alegre/ RS, 2013.

TEASELL, Robert. Reabilitação do acidente vascular cerebral. In: SPENCE, J. David. BERNETT, Henry J.M. Acidente vascular cerebral: prevenção, tratamento e reabilitação. Tradução BURNIER, Jussara N.T. Ed. Artmed. Porto Alegre- RS, 2013.

\section{(c) $\mathbf{B r}$}

Este trabalho está licenciado com uma Licença Creative Commons - Atribuição 4.0 Internacional. 